\title{
Polarimetry of Binary Stars and Exoplanets
}

\author{
Karen S. Bjorkman \\ Ritter Observatory, Department of Physics \& Astronomy, University of Toledo, USA \\ email: karen.bjorkman@utoledo.edu
}

\begin{abstract}
Polarimetry is a useful diagnostic of asymmetries in both circumstellar environments and binary star systems. Its sensitivity to asymmetries in systems means that it can help to uncover details about system orbital parameters, including providing information about the orbital inclination. Polarimetry can probe the circumstellar and/or circumbinary material as well. A number of significant results on binary systems have been produced by polarimetric studies. One might therefore expect that polarimetry could similarly play a useful role in studies of exoplanets, and a number of possible diagnostics for exoplanets have been proposed. However, the application of polarimetry to exoplanet research is only in preliminary stages, and the difficulties with applying the technique to exoplanets are non-trivial. This review will discuss the successes of polarimetry in analyzing binary systems, and consider the possibilities and challenges for extending similar analysis to exoplanet systems.
\end{abstract}

Keywords. instrumentation: adaptive optics, instrumentation: interferometers, instrumentation: polarimeters, techniques: image processing, techniques: polarimetric, (stars:) binaries (including multiple): close, (stars:) planetary systems

\section{Introduction}

The most useful purpose for polarimetry is in concert with all of the other kinds of observational image processing techniques. Polarimetry often is a non-unique solution in many cases, but when combined with spectroscopy, photometry, and interferometry it adds an extra dimension to what we can learn about all kinds of astronomical objects. I will review some of the basics about polarimetry, including the polarizing mechanisms in astronomy and different types of polarimetry measurements as applied to close binary stars and exoplanets. To begin, light is an electromagnetic wave and it oscillates. If we can measure the direction of that oscillation then we can measure its polarization. Every individual photon has a particular polarization. First, you need a source to polarize the light in a particular direction. Most of the light we see is not polarized, or at least it's just randomly polarized, so we measure no net polarization. If we have a system where the polarization has been preferentially selected for in one direction, then when we measure even an unresolved system we see a net polarization in the light from that system. An analyzer is needed to collect the information. This is often a half-wave plate that we put into a spectrograph, or an imager, to measure the degree of polarization, i.e., the amount of polarization and the position angle of the polarization. Both of these are critical bits of information.

An extremely useful tool for studying binary stars is called a $\mathrm{Q}-\mathrm{U}$ diagram, which is simply a plot of the Stokes U vs. Stokes Q parameters in the data that we've measured. The total intensity, I, is the sum of all the light that we see, including all light that is polarized in one direction plus all the light that is polarized in a perpendicular direction to that. If we take the difference between the polarization in one direction and the orthogonal polarization, we get the Stokes $\mathrm{Q}$ parameter, $\mathrm{Q}=\mathrm{P} \cos (2 \theta)$, where $\mathrm{P}$ is the 

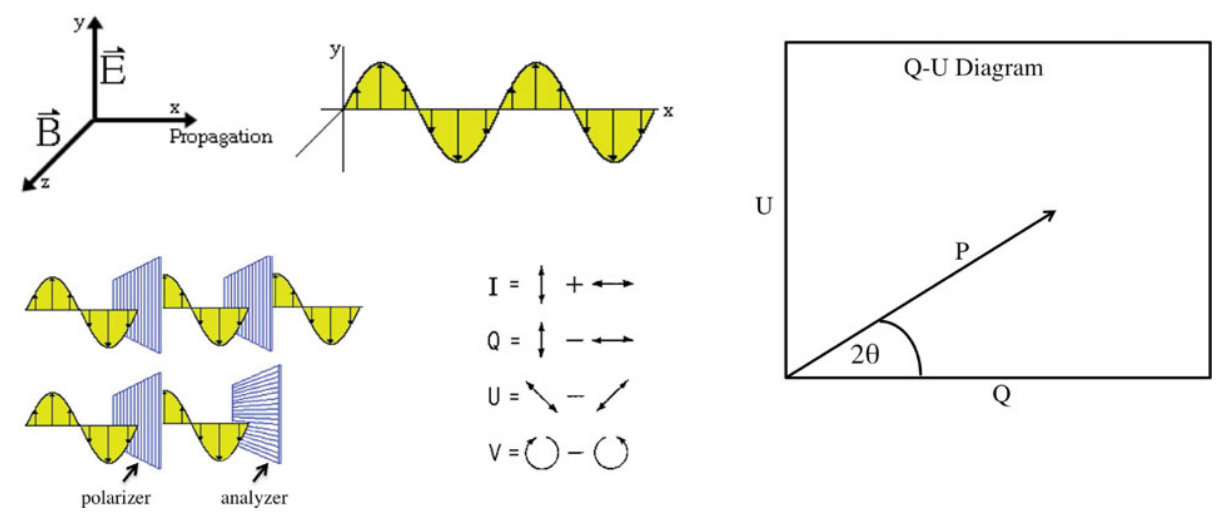

Figure 1. Basics of Polarization (left) and the Q-U Diagram (right)

polarization and $\theta$ is the position angle. The Stokes $U$ is essentially 45 degrees away, and again these two are orthogonal. This gives rise to the Stokes $\mathrm{U}$ parameter, $\mathrm{U}=\mathrm{P} \sin (2 \theta)$. So the polarization, $\mathrm{P}=\left(Q^{2}+U^{2}\right)^{1 / 2}$, and the position angle is $\theta=0.5 \arctan (U / Q)$. The polarization is really the length of this vector; if we have zero polarization at the origin $(0,0)$, we have no net polarization, and so we have $\mathrm{P}=0$ and no vector. But when we have some net polarization in a preferred direction, then we will measure some level of polarization $\mathrm{P}$ at some position angle which is $2 \theta$ on the sky. Typically in stellar systems the value of $\mathrm{P}$ can be as low as several tenths of a percent. That's because the star which is providing the light which is being scattered or polarized is also emitting light which does not get scattered, which dilutes the polarized light. So, you get very low levels of polarization in stellar polarimetry typically. In obscured sources, for example in brown stars or in AGNs, you can get polarization levels that are quite high (e.g., 20\% to 50\%) because the central source is obscured and you don't get any direct light that hasn't been scattered. You may wonder about the $2 \theta$ factor; that's simply because polarization in one direction is indistinguishable from polarization in the opposite direction; essentially because $0^{\circ}$ and $180^{\circ}$ produce the same results.

\section{Polarizing Mechanisms}

The list of polarizing mechanisms consists of (1) electron scattering, (2) dust scattering, (3) interstellar dichroic extinction, (4) thermal emission by aligned elongated dust grains (important in IR), (5) magnetic fields (circular polarization), and (6) line scattering.

Certainly electron scattering will polarize light, but if the scatterers are all symmetrically distributed, spherically distributed, as we'll see, the net polarization in an unresolved system will be zero because it will all average out.

We can have dust scattering; that is scattering off of dust grains. If those dust grains are spherically symmetric, they will not produce polarization. If they are elongated, as we think many dust grains are typically aligned because of the galactic magnetic field or stellar magnetic fields, they can produce a preferred orientation of the polarization. So, polarization is really a probe of asymmetries in many systems.

Interstellar dichroic extinction is produced when interstellar dust grains polarize light. It is unfortunate that many people think interstellar dust just gets in the way of our intended astronomical sources. In fact, one of the most difficult challenges in using polarimetry is being able to disentangle the contribution to the net polarization that you measure from the interstellar medium compared to the source that you're trying to study. 
So you have to be able to do a careful job of removing the contributions by the interstellar medium if you want to know what's actually happening in the your intended source. That can be a little tricky sometimes. Thermal emission by aligned elongated dust grains is also important. This is particularly important in young stars where there are large dust envelopes that may be affected by magnetic fields, and there is extra emission from the dust. Magnetic fields are a major component in polarization. In fact, circular polarization, the Stokes V parameter, is typically used to measure magnetic fields or to attempt to measure magnetic fields in stars where the Zeeman splitting is not strong enough to be measured. Usually this is the case in hot stars where the lines are broadened because of rotation. So, circular polarization is a very nice diagnostic of magnetic fields in stars, particularly in hot stars. The final polarizing mechanism is line scattering. Although many of us like to assume that spectral lines are unpolarized, in fact they may not be. We should be aware that there are processes in the line production that could polarize light. The models so far are relatively simplified and don't include line scattering polarization for the most part; so this mechanism is being actively worked on because we will need it in order to interpret the data accurately.

\section{The Effect of Asymmetries}

Here are some examples of how the asymmetries of the scatterers (e.g., electrons, dust grains, etc.) may affect what you see. Where polarimetry can really play a role is in systems they are far away, or too faint, so we can't resolve them directly yet. We have heard that interferometry and tomography are ways to begin to resolve these systems. Polarimetry is a way to analyze the asymmetries and the directionality of a system without actually being able to resolve it. I always find it amazing that I can tell you the position angle of a disk around a star when I can't even resolve the disk, even with interferometry or tomography. So, it is a powerful technique that we can exploit when we're trying to look for systems that are too far away to resolve.

Let us assume the very simple case of an unresolved system with a large number of electrons surrounding it in a circumstellar envelope; with the electrons uniformly distributed in density (no clumps) and uniformly distributed about the star (Fig. 2, left image). If light from the star scatters an electron, it becomes polarized and the direction of the polarization is perpendicular to the central plane (i.e., to the line between the source and the scatterer, for electron scattering). We can map put the polarization if the source is resolved. However, if the source is not resolved, we essentially sum over the entire envelope; hence the net polarization that we measure is zero, since there are as many vectors in one direction as in the perpendicular direction, so they cancel each other out. So the net polarization in a perfectly spherical system would be zero.

In reality, the systems are not spherical. If we have blobs, we may have scattering into different blobs, we may have different densities in these blobs, and we may have different sizes and orientations. In the case of an unresolved system, the net polarization will depend on the relative contribution from each of the blobs doing the scattering (Fig. 2, middle image). This gets complicated because we have to include more parameters, like the blob distribution or the distribution of the scatterers. If you have a disk or a jet (Fig. 2, right image), then all of the scatterers are confined to a relatively thin disk (e.g., accretion disks or Be star disks). In this case, the net polarization is perpendicular to the disk or to the jet. So, if we measure the polarization of an unresolved star, we can still measure the position angle of the disk from the polarization.

This theoretical prediction was confirmed in a simple test case in which polarmetric observations were combined with interferometric observations of a resolved disk. We 


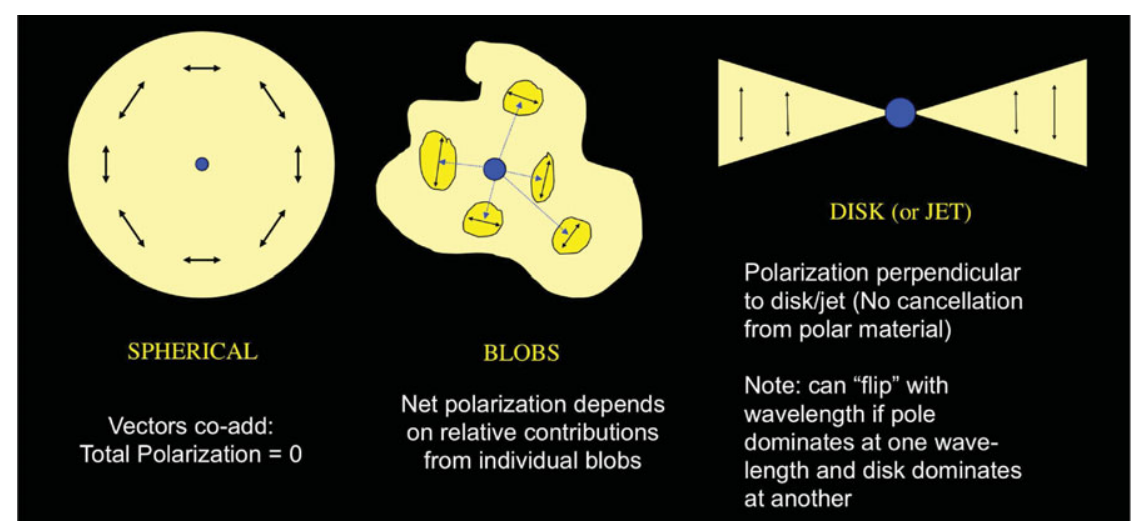

Figure 2. Effect of asymmetries in unresolved systems. The polarization at each point is perpendicular to the radius vector.

found that the position angle derived from the polarization was exactly in agreement with the interferometry image. Hence, the prediction holds true. This is very useful because it means that we can determine position angles of disks that we cannot resolve. We have discovered that the position angles can be wavelength dependent if the scatterers near the pole dominate at one particular wavelength while the disk dominates at another wavelength. We see this effect in stars with thick stellar envelopes, like Herbig Ae/Be stars.

\section{Common Polarization Measurements}

There are several polarimetric measurements in common use, including (1) broadband photo-polarimetry (UBVRI etc), (2) imaging polarimetry, (3) spectropolarimetry, and (4) circular polarimetry of spectral lines. The simplest measurement is broadband photopolarimetry, which is essentially photometry using a polarimetric analyzer in conjunction with a photometric filter. For example, the polarization of a star can be measured at the different wavelengths in the UVBRI bands to provide the wavelength dependence for the polarization.

Imaging polarimetry is possible if the analyzer (e.g., a half wave plate) is placed in front of an imaging device. For example, if we are studying many stars in a cluster, we can take a direct image of that cluster or we could place a polarizing analyzer in the light path. By doing so we measure both the ordinary and extraordinary polarization for each star in the field; hence with imaging polarimetry we can measure many stars at once. Another application is in resolved sources, e.g., young stars. If the sources are nearby, we can map out the polarization distribution within the young star envelope. This can be very interesting in terms of understanding the structure within that envelope. Spectropolarimetry is much more complicated because it involves spectroscopy with a polarizing analyzer. This procedure creates a spectrum of the ordinary polarized light and a second spectrum from the extraordinary polarized light. By comparing the two spectra we can measure the polarization as well as the polarization as a function of wavelength, just as we do in spectroscopy. Instead of just measuring the flux, we simultaneously measure both the flux and the polarization at each wavelength. At this conference, we have been talking about the challenges of observing bright stars and faint stars and the need for bigger telescopes; spectropolarimetry is even more delicate because it is 
clearly a photon-starved, photon-limited technique, and it will be quite challenging to get measurements at the $1 \%$ level.

Circular polarimetry of magnetically-sensitive spectral lines is used to get information about the magnetic field by measuring the Stokes V parameter in cases where the Zeeman splitting is weak.

\section{Applications of Polarimetry}

Imaging polarimetry was used to observe many stars at once in clusters in the Large Magellanic Cloud (e.g., NGC 1948). This was a program to identify unresolved disk systems in the LMC (Wisniewski et al. 2005). The procedure creates a double image for every star because the polarizing analyzer is placed in front of the image. One is the extraordinary and the other is the ordinary image. Instead of just doing photometry on one image of a star, we do photometry on both of images and then compare them. Then, we rotate the polarizing analyzer and take another image, and repeat the process for additional rotations of the polarizing analyzer, until we get a measurement of the polarization for each star in the field. The analysis is more complicated when the field is crowded because of confusion with overlapping images; instead higher spatial resolution techniques are needed to separate the cores of crowded clusters. However, the procedure works well in the general field far from the cluster core.

An amazing example of a resolved source is FS Tau, a $0 .{ }^{\prime \prime} 2$-separated classical T Tauri binary system, taken with the Coronagraphic Imager with Adaptive Optics (CIAO) on the Subaru Telescope and superimposed on the HST image (Hioki et al. 2011). This is a young stellar object in a binary system. The polarization is represented by little vectors across the image, and the orientation of the vector gives the direction of the polarization at that point in the field. The length of the vector indicates the strength of the polarization; the longer the vector the larger the polarization. In FS Tau, there is a real centro-symmetric pattern, similar to what is shown in Fig. 2 (left frame). Hence, the scatterers are distributed about the star in a very particular way. FS Tau is actually a highly-polarized object because the central source (the star) is obscured in the image. It was possible to use the HST to measure the polarization in this case because of its strength. It is not commonly known that the Hubble Space Telescope originally had polarizing optics in it; but the COSTAR upgrade affected the instrumental polarization and it became very difficult to make measurements at low polarization levels. However, it is still quite useful for high polarization levels.

An nice example of spectropolarimetry is shown in Wood, Bjorkman, \& Bjorkman (1997) for the classical Be star $\zeta$ Tau, which is also a binary (see Fig. 3). This figure looks like a spectrum, but it is a measurement of polarization as a function of wavelength from the ultraviolet to the infrared $(10,000 \AA)$. It shows the polarization Balmer jump, the Paschen Slope, the Paschen Jump, and so on. There are relatively few polarization observations of objects in the ultraviolet. HST could make these observations for a while, and it can still do so. However, the Wisconsin Ultraviolet Photo-Polarimeter Experiment (WUPEE) was the only dedicated instrument even flown, other than balloon flights, to do broadband spectropolarimetry in the ultraviolet. WUPEE flew twice on the Space Shuttle during the ASTRO-1 (1990) and ASTRO-2 (1995) missions, and it is the only current source of spectropolarimetry in the ultraviolet. WUPPE collected UV spectropolarimetry and spectra for 121 objects.

The appearance of the WUPEE spectra were surprising because the models predicted that the polarization would rise towards the UV, but this was not the case. The explanation was that the polarized light, which has been scattered in the disk, still has 


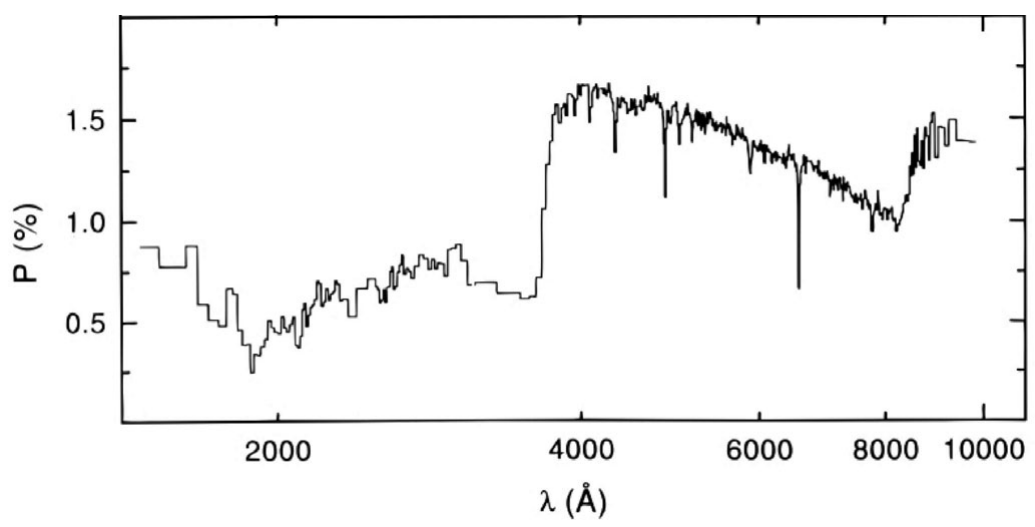

Figure 3. Spectropolarimetry of $\zeta$ Tau before correction for interstellar reddening and interstellar polarization taken from Wood, Bjorkman, \& Bjorkman (1997) (their Figure 3).

to get out of that disk. So, as it travels through the disk, either before or after it gets scattered, some of that light gets absorbed by the opacity in the disk. So the spectrum of the disk can be obtained directly if you look at the polarized light, even though the disk is unresolved in most cases with interferometry. We also get some physics from the polarimetry because we can measure opacities and temperatures and structures of the disk from the polarized spectrum.

The photon counting error bars and systematic errors associated with the data are very small. Zeta Tau has very little interstellar polarization in its direction, so no correction was needed for those effects. The effect of the lines can clearly be seen in Figure 3 (e.g., Balmer lines, Paschen jump), so the polarization mimics the hydrogen opacity, and line blanketing from the disk itself is revealed in the UV part of the spectrum (e.g., in the Fe III and Fe II lines). This is just an example of the applications if you have bright enough stars and high enough signal to noise.

\section{Polarimetry of Binaries}

A lot of the early work on the polarimetry of binaries was done in the 1960s and 1970s. The most commonly used models are by Brown, McLean, \& Emslie (1978) and Rudy \& Kemp (1978). In these models, they assume two stars in a circular orbit surrounded by a co-rotating envelope that was optically thin and undergoing single scattering. This is a simple analytical approach, but it predicted that Q-U diagrams could diagnose both inclination angles and eccentricities for binary orbits. Brown, McLean, \& Emslie (1978) showed that the binary traces out a loop or a double loop in the Q-U diagram as the phase advances; and this behavior depends on the inclination and eccentricity of the orbit.

We know that binary stars, especially close binaries, are more complex than described in these models. Interacting binaries contain mass transfer streams, hot spots where the stream falls into the accretion disk, the disk around the gainer star, and an asymmetric donor star. Each of these components represents an asymmetry that contributes to the net polarization. There have been several attempts to disentangle all of these parts using spectropolarimetry (e.g., Hoffman et al. 1998, Hoffman et al. 2003; for $\beta$ Lyr). There are changes in the polarized flux as a function of wavelength (the spectropolarimetric flux) at phases before, during, and after primary and secondary eclipse. These changes 
can be used to diagnose some of the physics. Several models use the UV to look at the jet, and use the optical to look at the disk, and then combine the separate results; so it is quite challenging. Barbara Whitney and collaborators have also performed Monte Carlo modeling of optically thick disks. The latest models now include the hot spot (e.g., Lomax \& Hoffman 2011; $\beta$ Lyr).

Polarimetry of other binary systems include the pre-main-sequence binaries (e.g., Manset \& Bastien 2000, Manset \& Bastien 2001a, Manset \& Bastien 2001b); Wolf-Rayet binaries (e.g., St.-Louis et al. 1987, St.-Louis et al. 1988); and Algols (e.g., Pfeiffer \& Koch 1977).

\section{Polarimetry of Exoplanets}

Exoplanets are like little binaries. Sara Seager and her collaborators (Seager, Whitney \& Sasselov 2000) predicted the level of polarization you might expect from scattering off the atmosphere of an exoplanet; and their levels were on the order of $10^{-6}$. This was not at a level that we could measure with current polarimeters, so this really discouraged many people from trying to apply polarimetry to exoplanets. However, Berdyugina et al. (2011) have claimed a detection at about a level of $10^{-4}$. There is still some discussion about this result, so they collected multiwavelength UBV observations and it seems that the detection is real.

Another method is to search for limb polarization during a transit (e.g., Davidson et al. 2010). Carciofi \& Magalhaes (2005) predicted various polarization levels that could be observed for these limb transits of exoplanets, and they are at the level of $10^{-6}$. However, this limit can be increased to around $10^{-4}$, which is in fact observable.

To detect stellar polarization at levels of at least $10^{-4}$ requires extremely high $\mathrm{S} / \mathrm{N}$. The number is very very big. It requires that we use large telescopes or observe bright stars, or preferable both, especially if you want to do spectropolarimetry. Differential polarization, like differential photometry, can help because absolute calibration is not needed but the instrumental polarization has to be very carefully calibrated and removed, and it has to be stable. It is important to remove the interstellar polarization, especially for time variable phenomena, and it is not an easy thing to do.

\section{Future Directions}

Polarimetry can now be used to detect non-transiting exoplanets at all orbital inclinations. There is hope that we may even be able to use spectropolarimetry to measure the composition of the atmospheres of exoplanets, and there are polarimeters being designed for large telescopes that may achieve this goal. Work is now being done to predict the spectropolarimetry of an exoplanet with light scattering from a star off the atmosphere for a hot Jupiter model. In this case, the polarized spectrum can essentially serve as a surrogate for the spectrum of the atmosphere of the planet. It will take a lot of effort to achieve this kind of detection. Finally, Q-U plots are useful tools because they provide the same kinds of diagnostics for exoplanet systems as they do for binary systems; so we can derive parameters like orbital inclinations and eccentricities for them.

\section{Discussion}

R. Wilson: About 15 to 20 years ago I made a computational model for time-wise variability of polarization in close binaries, including limb polarization and disk polarization. That model remains the only one that does the problem quantitatively (both direct and 
inverse. It solves for the parameters by least squares, and does it without unnecessary intermediaries such as Fourier series fitting). The model would have been developed further, but there were no more observations to process after the J. Kemp data on Algol. Why has there been an emphasis on surveys to the almost complete exclusion of polarization curves for individual binaries? Any limitation due to photon noise should apply equally to surveys and individual variation.

K. BJORKMAn: Thank you for reminding us of these existing models. You are correct that what is needed is more time-monitoring observations of polarimetry for these systems. Unfortunately, as we know, it can be difficult to obtain sufficient observing time for monitoring observations. There are some databases of existing polarimetric data (e.g., the HPOL data available through the NASA MAST web site); however, additional data and polarimetric instruments are still needed.

R. WILSON: Why has there been an emphasis on surveys while time-wise variations of basic simple systems are ignored?

K. BJorkman: Long-term monitoring is being done on small telescopes. For example, most of the HPOL data were obtained on a $1 \mathrm{~m}$ telescope at Wisconsin, where we monitored for 15 years and we had students observing in a Q mode. We had a lot of data for particular sets of stars, but you are right that we need to do a lot more monitoring. The time variability is much more crucial than the surveys, in a sense.

\section{References}

Berdyugina, S. V., Berdyugin, A. V., \& Piirola, V. 2011, A\&A, submitted

Brown, J. C., McLena, I. S., \& Emslie, A. G. 1978, A\&A, 68, 415

Carciofi, A. C. \& Magalhães, A. M. 2005, ApJ, 635, 570

Davidson, J. W., Bjorkman, K. S., Magalhaes, A. M., Carciofi, A. C., Bjorkman, J. E., Seriacopi, D. B., \& Wisniewski, J. P. 2010, BAAS

Rudy, R. J. \& Kemp, J. C. 1978, ApJ, 221, 200

Hioki, T., Itoh, Y., Oasa, Y., Fukagawa, M., \& Hayashi, M. 2011, PASJ, 63, 543

Hoffman, J. L., Nordsieck, K. H., \& Fox, G. K. 1998, AJ, 115, 1576

Hoffman, J. L., Whitney, B. A., \& Nordsieck, K. H. 2003, ApJ, 598, 572

Lomax, J. R. \& Hoffman, J. L. 2011, Bull. Soc. Roy. Sci. de Liege, 80, 689

Manset, N. \& Bastien, P. 2000, AJ, 120, 413

Manset, N. \& Bastien, P. 2001a, AJ, 122, 2692

Manset, N. \& Bastien, P. 2001b, AJ, 122, 3453

Pfeiffer, R. J. \& Koch, R. H. 1977, PASP, 89, 147

Seager, S., Whitney, B. A., \& Sasselov, D. D. 2000, ApJ, 540, 504

St.-Louis, N., Drissen, L., Moffat, A. F. J., Bastien, P., \& Tapia, S. 1987, ApJ, 322, 870

St.-Louis, N., Moffat, A. F. J., Drissen, L., Bastien, P., \& Robert, C. 1988, ApJ, 330, 286

Wisniewski, J. P., Bjorkman, K. S., Magalhes, A. M., Bjorkman, J. E., \& Carciofi, A. C. 2005, $A S P-C S, 337,333$

Wood, K., Bjorkman, K. S., \& Bjorkman, J. E. 1997, ApJ, 477, 926 